\title{
La obra coronada en la fortificación de Puerta Tierra (Cádiz) a través de la cartografía urbana del siglo XVIII
}

The crownwork fortification of Puerta Tierra (Cadiz) through the eighteenth century urban maps

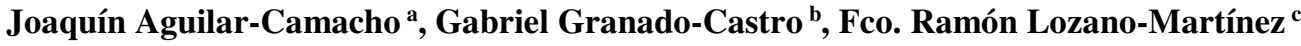 \\ Departamento de Ingeniería Gráfica - Universidad de Sevilla, Seville, Spain \\ a jacmpit@us.es; ${ }^{\text {b }}$ ggranado@us.es; ${ }^{\text {c }}$ ramonlozano@us.es
}

\begin{abstract}
Most of the walls of Cádiz is mainly due to the works carried out on the seventeenth century. During this period the defenses of the Puerta Tierra wall -the city's only terrestrial communication point- were expanded with the incorporation of new defensive elements, such as the so-called "crownwork". However, it was during the eighteenth century when this defensive element acquired its last configuration, which did not correspond to the last planned project.

This paper offers a vision on the various projects make by military corps of engineers for the remodeling of the Puerta Tierra wall. For this purpose we relies on the historical maps of the city corresponding to the eighteenth century. Also, it is analyzed how during this century its physiognomy evolved until the disappearance of the crownwork.
\end{abstract}

Keywords: Fortification, historical cartography, military engineer, defensive elements.

\section{Introducción}

Desde principios de la Edad Moderna el comercio con las Indias cernió sobre Cádiz la amenaza real de continuas incursiones berberiscas encabezadas por el corsario Khaireddin Barbarroja y apoyadas por el sultán turco Solimán. Las obras para su fortificación no tardaron en iniciarse hacia el año 1530, con la participación en su diseño de los más prestigiosos ingenieros militares italianos.

En 1596, cuando los tercios españoles se disponían a invadir las islas británicas, Isabel I de Inglaterra ordenó atacar la flota de la Armada Española, fondeada en Cádiz. El asalto tuvo lugar el 30 de junio, dejando malparada la ciudad -incendiada y arrasada-. La incursión puso en evidencia la eficacia de las defensas construidas hasta la fecha.
Pese a ello, Felipe II apostó por su reconstrucción, el refuerzo de las defensas existentes y la fortificación de los puntos estratégicos de la plaza, la bahía y el litoral. Comienza así, a principios del siglo XVII, una nueva y larga etapa de intensa actividad urbanística y defensiva para la ciudad y su entorno, que vivirá el período de mayor esplendor durante el siglo XVIII, gracias al florecimiento de la actividad comercial.

\section{Antecedentes}

Las fuentes históricas no permiten establecer la fecha exacta en la que se construyó por primera vez un elemento defensivo para proteger el único acceso terrestre de Cádiz, conocido como 
frente de tierra o de levante -también como Puerta Tierra-.

En la primera imagen conservada de la ciudad (Fig. 1), es posible identificar una elemental configuración del genuino frente de tierra a principios del siglo XVI, emplazado en la cerca amurallada de la antigua villa medieval.

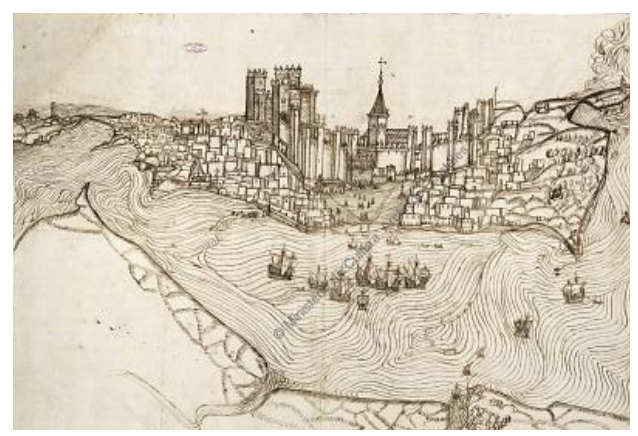

Fig. 1. Dibujo de la ciudad de Cádiz sobre un postigo, Anónimo (Archivo General Simancas, MPD-25-047).

El paulatino crecimiento de la ciudad hacia levante propició un nuevo emplazamiento y configuración de este frente, conformado por un muro de tierra con puerta de entrada y salida en el centro (puerta del muro). Este sencillo elemento defensivo pretendía proteger de un posible ataque por tierra al arrabal de Santa María, uno de los dos arrabales formados fuera de la primitiva cerca de la villa. De su utilidad, como elemento defensivo, recelaba en junio de 1529 el corregidor de la ciudad (Fernández Álvarez, 1966, p. 429).

Durante el siglo XVI, a raíz de la apertura al comercio indiano, la ciudad redobló los esfuerzos por mejorar su estado defensivo frente a las continuas incursiones de corsarios y berberiscos. La intervención de diversos ingenieros italianos -entre los que destacan las figuras de Giovanni Battista Calvi, Giacomo Paleari Fratino y Tiburzio Spannocchi-, contribuyó a configurar su traza con las últimas innovaciones del sistema defensivo italiano mediante frentes abaluartados. Luis Bravo de Laguna, máximo responsable del Consejo de Guerra de Felipe II, fue encomendado, en 1577, para elaborar un exhaustivo estudio sobre el estado defensivo de la costa meridional andaluza. Estando en Cádiz, informó que consideraba el muro de tierra una buena muralla, en la que se construían dos baluartes, los de Benavides y San Roque. En 1578, el Consejo de Guerra ordenó al corregidor de Cádiz la puesta en defensa de estos baluartes y la construcción de un foso delantero (Fernández Cano, 1973, pp. 20-22).

En 1587, se desencadenó la guerra con Inglaterra, tras el apoyo inglés a los Países Bajos en el conflicto de Flandes. En ese mismo año, tuvo lugar una primera incursión inglesa sobre la ciudad, comandada por el corsario Francis Drake, que terminó con la destrucción de gran parte de la Armada Española anclada en la bahía de Cádiz.

El ataque de 1596 supuso un claro punto de inflexión en el devenir histórico de Cádiz. Tras el mismo, Felipe II llegó a plantearse el abandono de la ciudad en su actual emplazamiento y su reconstrucción en las inmediaciones del Puerto de Santa María (Abreu, 1866; Usherwood, Usherwood, 2001). Finalmente, Felipe II descartó el desmantelamiento de la ciudad, inclinándose por su reconstrucción y fortificación.

Tras el asalto inglés, surgieron diversos proyectos para su defensa -destacan los de Cristóbal de Rojas, Pedro Velasco y Añasco y de Luis Fajardo-, que fueron confrontados con la propuesta de Spannocchi, previa al ataque. Entre las obras finalmente aprobadas, en el año 1597, se encontraba la conclusión de los baluartes de Benavides y San Roque, iniciados en el frente de tierra por Fratino (Fernández Cano, 1973, p. 40; Ruiz Nieto-Guerrero, 1999, p. 42).

En los sucesivos años el perímetro de la ciudad se fue amurallando progresivamente, aflorando en el mismo baluartes, castillos y cortinas, que lograron transformarla en un auténtico fortín cuya efectividad desmostró el fallido ataque anglo-holandés de 1625-. El grueso del amurallamiento de Cádiz se debe, fundamentalmente, a las obras realizadas durante el siglo XVII (Bustos Rodríguez, 2008, p. 419).

Además, se ampliaron las defensas del muro de tierra con la construcción de nuevos elementos 
poliorcéticos (Fig. 2). Concretamente, tras el asalto inglés de 1596 se procedió, en años sucesivos, a la construcción de distintos elementos defensivos. Primero se derribó el viejo muro medieval, quedando con menor altura otra cortina más reciente. Se edificó el revellín y el foso inicial para defender el vano de acceso a la ciudad y se concluyeron las obras de los dos baluartes contiguos -Benavides y San Roque-, dotándolos de casamatas y piezas artilladas.

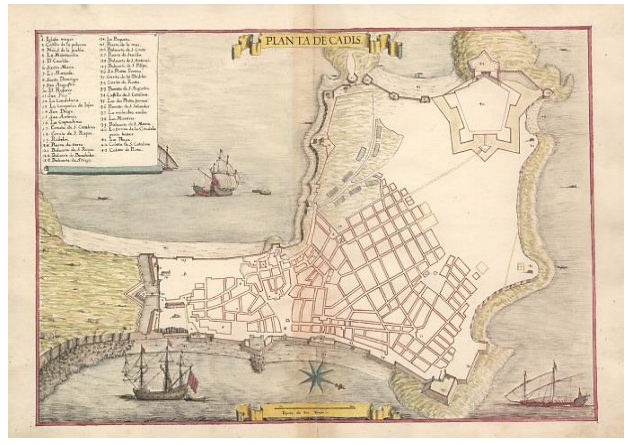

Fig. 2. Planta de Cádiz, 1650-55, Leonardo de Ferrari (Atlas Heliche. Archivo Militar de Estocolmo).

En 1602, el Consejo de Guerra aprobó la construcción de un castillo-ciudadela en el Frente de Tierra, según proyecto de Spannocchi. La obra quedaría detenida apenas iniciada, no llegando a construirse debido a la falta de fondos y a considerarse más conveniente cercar toda la ciudad (Fernández Cano, 1973, pp. 90-91).

Posteriormente, hacia 1625, la profundidad del foso fue aumentada. Más adelante, a partir del año 1656, se acometió el perfeccionamiento del sistema defensivo del frente de Puerta Tierra, con la construcción de la llamada "obra coronada" delante de la muralla -concluida en 1671-, por iniciativa del conde de Molina (Fig. 3). Los restantes años del siglo XVII no conllevaron un progreso significativo en las obras de fortificación de la plaza, observándose cierto desinterés en las mismas por la confianza en la eficacia de sus defensas, demostradas tras el fallido ataque de 1625 (Fernández Cano, 1973, pp. 51, 75, 95, 121-122).

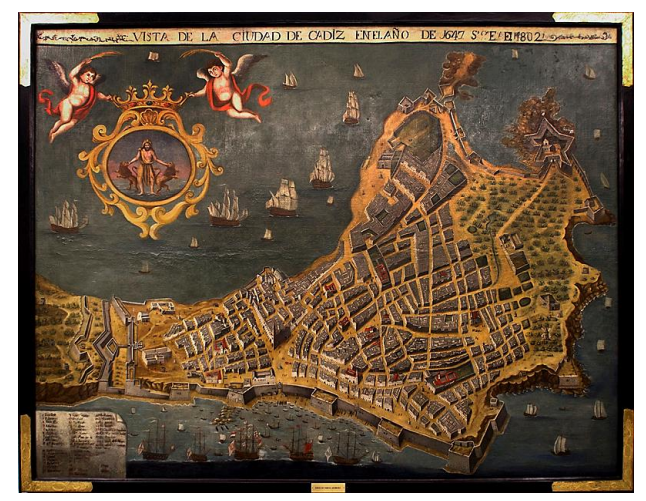

Fig. 3. Vista Aramburu, Cádiz, s. XVII, anónima (Museo de las Cortes de Cádiz).

\section{Proyectos para su remodelación y mejora durante el siglo XVIII}

A lo largo del siglo XVIII el frente de tierra alcanzó su fisonomía definitiva. Los incesantes conflictos bélicos a los que se enfrentó España durante la centuria; la localización en Cádiz de la cabecera en la ruta comercial con las Indias (1717); la imperiosa necesidad de convertir la ciudad en un reducto inexpugnable; el continuo perfeccionamiento de los sistemas defensivos y la influencia del Corps du génie francés y sus tratadistas, con Sébastien Le Prestre -marqués de Vauban- como referente, afanó la labor del recién creado cuerpo homólogo español (1711) en su defensa y consolidación como plaza fuerte, propiciando diversos proyectos de remodelación para la mejora defensiva de esta fortificación.

La relevancia alcanzada por la ciudad a principios del siglo XVIII, permite afrontar una centuria rica en mapas y planos, convirtiendo a Cádiz en una urbe privilegiada en este tipo de fuente documental. Se tratan, fundamentalmente, de plantas urbanas sobre la ciudad asociadas consubstancialmente a la labor de intervención de los ingenieros militares en las tareas defensivas y de fortificación de su perímetro, y que han legado un valioso testimonio acerca de las numerosas propuestas de configuración del frente de tierra proyectadas en este período. 


\subsection{Remodelación de los glacis en la obra coronada y en la trinchera del foso}

La primera propuesta de intervención a lo largo del siglo XVIII, la encontramos en una representación francesa de la ciudad, titulada Ville de Cadix, realizada en 1707 por Picot (Fig. 4). Esta planta muestra un proyecto -en color amarillo- para la construcción de nuevos terraplenes en la fortificación de Puerta Tierra -delante de la obra coronada y de la trinchera que antecede al foso del hornabeque-.

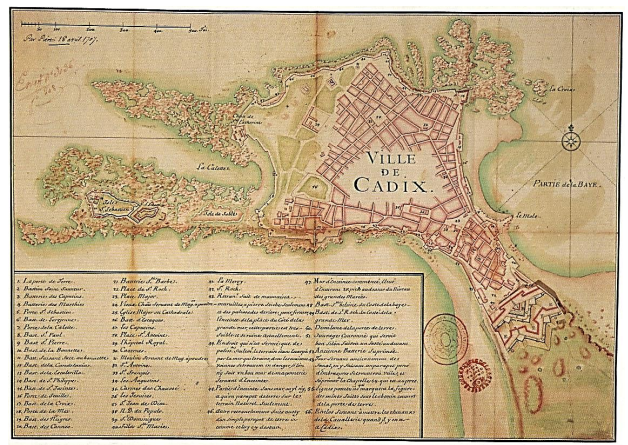

Fig. 4. Ville de Cadix, 1707, Picot (Bonet Correa, 1991, p. 57).

A finales del siglo XVII, la arena acumulada por el temporal de levante llegó a cegar el foso y las cortinas de la muralla, malogrando la función defensiva de toda la fortificación. Antes de remodelar los terraplenes se llegaron a probar diversas soluciones temporales -parapetos con vegetación y antepechos de madera-, que fracasaron (Fernández Cano, 1973, p. 97).

El mismo proyecto aparece representado en color amarillo en un plano de 1713, de Diego Luis Arias (Fig. 5), con una ligera modificación. En este caso se proyectaron dos baterías en sendos flancos de la obra coronada (baterías de la Cruz y de las Bacas). En la leyenda del plano se estima el costo de la remodelación del Frente de Tierra en algo más de 330000 reales de vellón. Estas obras, junto a otras del perímetro defensivo de la ciudad, se encontraban pendientes de ejecutar -por falta de medios-, según se desprende de la leyenda.

En una planta anónima (Fig. 6), que por la configuración de su trama y el contenido de la leyenda podemos datar entre los años 1723 y 1724, se representa -ya ejecutado- el nuevo terraplén correspondiente a la obra coronada, según el proyecto mostrado en la planta de Picot. Para su construcción fue preciso derribar la pequeña ermita de Santa Elena, construida en 1640 y situada en las inmediaciones. De ella tomaría nuevo nombre el baluarte que, hasta la fecha, se había llamado de Benvaides (Fernández Cano, 1973, p. 97). El proyecto de nuevo terraplén delante de la trinchera del foso no aparece representado en este plano.

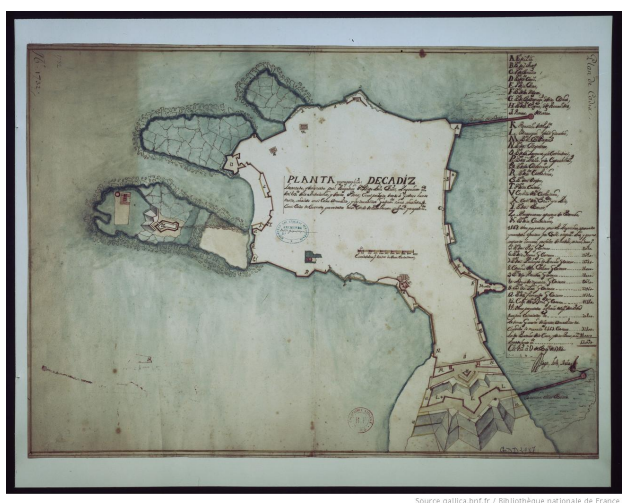

Fig. 5. Planta yconogrphica de Cadiz, 1713, Diego Luis Arias (Bibliothèque nationale de France, GE DD2987-1732).

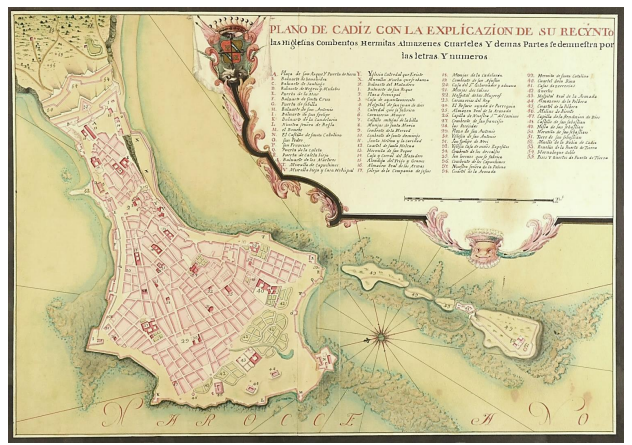

Fig. 6. Plano de Cadiz con la Explicazion de su Recynto, 1723-24, anónimo (Archivo General Militar de Madrid, AT-194/28).

\subsection{El proyecto de Verboom e Ignacio Sala}

Jorge Próspero de Verboom, ingeniero flamenco, discípulo de Sébastien $L e$ Prestre, Señor de Vauban; hijo de Cornelio Verboom, Ingeniero Mayor de los Países Bajos; 
fue, además, el fundador (año 1711) del Cuerpo de Ingenieros Militares español, durante el reinado de Felipe V. Trabajó activamente en las obras para la defensa y fortificación de Cádiz, entre finales del primer cuarto de siglo y principios del segundo.

En 1724, Verboom dirigía las obras de fortificación de Cádiz. Elaboró un proyecto para la remodelación del Frente de Tierra (Fig. 7) con objeto de mejorar su funcionalidad defensiva. Sin embargo, la propuesta no fue original, pues reprodujo íntegramente un proyecto de remodelación francés redactado en 1710, en plena Guerra de Sucesión, probablemente por un ingeniero militar cuyo verdadero nombre $-\mathrm{D}$. par A. Bertau o Bertan o Bertaux- no ha sido posible esclarecer (Fig. 8).

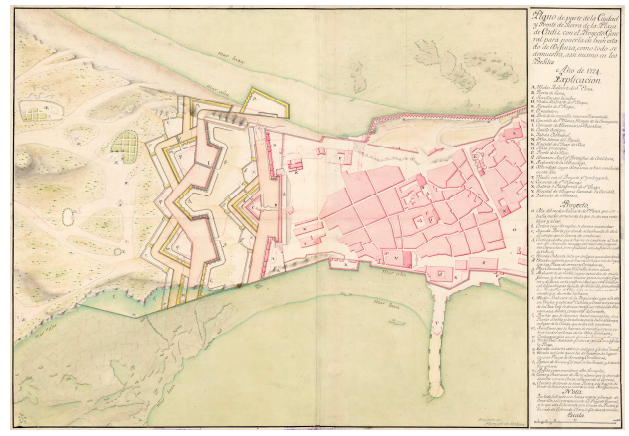

Fig. 7. Proyecto de remodelación de la fortificación de Puerta de Tierra, 1724, Verboom (Centro Geográfico del Ejército Ar.G-T.8-C.1-591).

El proyecto afectaba tanto a la obra coronada, como al actual revellín y al hornabeque.

En relación al primer elemento, preveía demoler su actual estrada cubierta, siendo reemplazada por una nueva más adelantada en la que se debían construir dos nuevos revellines para proteger las cortinas de la obra coronada; construcción de dos contraguardias en sendos extremos de la obra coronada; construcción de un semibaluarte destacado para defensa de la playa de Santa María.

Respecto al revellín adelantado del hornabeque, propone Verboom la construcción de una nueva estrada cubierta interior, con sendos revellines adelantados y nueva contraguardia alrededor del revellín original, para ampliar su capacidad. En cuanto al hornabeque, estaba previsto ensanchar sobre el foso el terraplén de la cortina central.

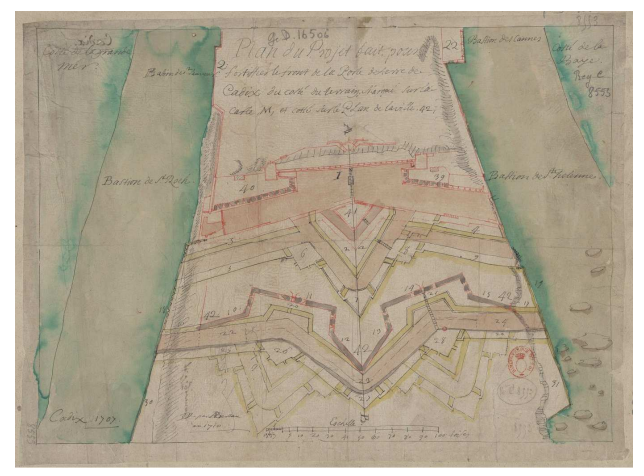

Fig. 8. Plan du Projet fait pour fortifier le front de la porte de terre de Cadix du costé du terrain, 1710, D. par A. Bertau (Bibliothèque nationale de France, GE D-16506).

Por último, contemplaba la ampliación de todo el flanco correspondiente al medio baluarte de Santa Elena, que se encontraba abierto y en ruinas, edificando sobre terreno ganado a la bahía. Tanto en la obra coronada como en el revellín original, se comtemplaban construir nuevos pasos con puentes levadizos para la salida y entrada a la ciudad.

Al frente del proyecto original de Verboom, aprobado por Felipe V en febrero de 1728, continuó Ignacio Sala, ingeniero director interino de la plaza desde diciembre de 1726 . Sala había trabajado activamente con Verboom en Cataluña y Aragón durante la década de 1710 (Capel-Sáez, et al., 1983, pp. 418, 422; CanoRévora, 1994, pp. 320, 371-375).

Uno de los últimos planos (figura 9) firmados por Pedro Borrás como ingeniero director de la plaza -cargo que ocupaba desde 1717 (CanoRévora, 1994, pp. 114-115)-, proyecta en 1726 una remodelación en las minas subterráneas de la fortificación de Puerta de Tierra. En el plano se especifican las minas que quedarán en desuso, las que se recuperarán y las nuevas que se habrán de ejecutar.

En este plano, aparece concluida la segunda estrada cubierta proyectada delante de la obra coronada. Sin embargo, su diseño difiere respecto al proyecto de Verboom aprobado en 
1728 (Fig. 7), por circunstancias que se desconocen. No fueron construidos los dos revellines adelantados a la obra coronada, según estaban previstos, y se introduce una segunda plaza de armas en la estrada cubierta, no prevista en el proyecto original.

En otro plano de abril de 1730 (Fig. 10), firmado por Ignacio Sala, aparece ya construida la primera estrada cubierta con sus plazas de armas, tal cual la proyectó Verboom alrededor del revellín adelantado al hornabeque. También se respresentan, en ejecución, la cimentación de la nueva contraguardia que se había de construir alrededor de este revellín y la ampliación del flanco lateral del medio baluarte de Santa Elena.

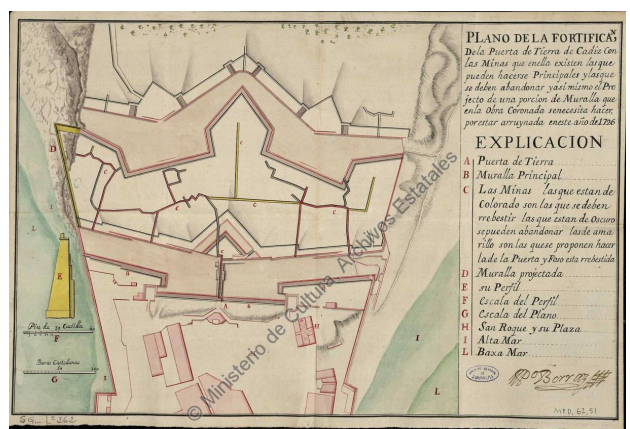

Fig. 9. Plano de la fortificación de la Puerta de Tierra de Cádiz, 1726, Pedro Borrás (Archivo General de Simancas, MPD-62-051).

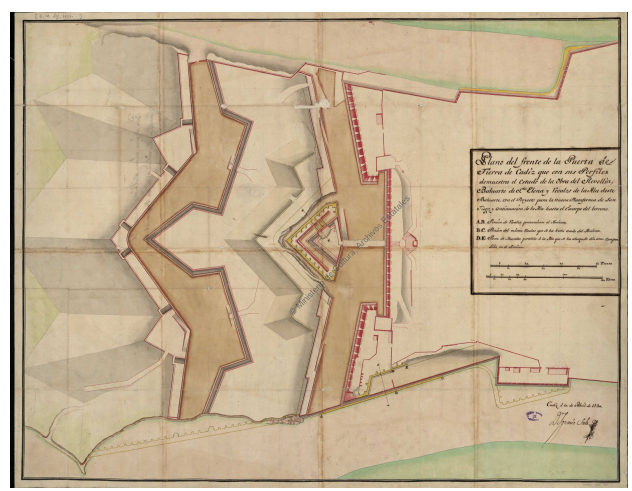

Fig. 10. Plano del frente de la Puerta de Tierra de Cadiz, abril 1730, Ignacio Sala (Archivo General de Simancas, MPD-21-075).

\subsection{Sustitución de la obra coronada}

Desde su aprobación en febrero de 1728, las obras de remodelación del frente de Puerta
Tierra avanzaban a buen ritmo bajo la dirección de Ignacio Sala, con la supervisión de Jorge Próspero Verboom.

Sin embargo, el mal estado de conservación en el que se encontraba la muralla de la obra coronada, con un elevado costo de reparación, propició la remodelación del proyecto original concebido por Verboom en 1724 (Fernández Cano, 1973, p. 124).

En septiembre de 1730, por indicación de Verboom, Ignacio Sala redacta una propuesta de remodelación alternativa que contemplaba la sustitución de la obra coronada. Su espacio quedaría ocupado por el glacis de la estrada cubierta proyectada delante del revellín del hornabeque. Además, la construcción prevista de una nueva contraguardia adelantada sobre este revellín, quedaría ampliada, a ambos flancos de la primera, con dos nuevas contraguardias. Para atender la defensa de estas dos nuevas contraguardias, el proyecto preveía modificar la geometría original del revellín principal, que pasaría de dos a cuatro flancos. Por último, el proyecto contemplaba la construcción de sendos orejones en los mediobaluartes de San Roque y Santa Elena, con el fin de mejorar la defensa del foso. La nueva propuesta conservaba la construcción de un medio baluarte destacado sobre la playa de Santa María (Fig. 11).

En 1737, las obras de esta última remodelación no habían comenzado aún. Se trabajaba, por entonces, en las obras necesarias para consolidar la ampliación de la fortificación de Puerta Tierra hacia el flanco de la bahía ${ }^{1}$. En 1739, había comenzado la construcción de la contraguardia del baluarte de Santa Elena; en 1740 la del baluarte de San Roque; y en 1741 la de la contraguardia central ante revellín del hornabeque $^{2}$, que avanzaban con lentitud por su complejidad (Fernández Cano, 1973, pp. 124125).

En 1745, Ignacio Sala introdujo de nuevo modificaciones en su proyecto de reforma del frente de Puerta de Tierra, que no llegarían a ver la luz. En ellas, ampliaba las dimensiones de la primera estrada o camino cubierto -el más inmediato al revellín del hornabeque- y proyectó minas y hornillos bajo el glacis de este primer 
camino. Además, contempló la construcción de una lengua de sierpe adelantada en el centro de la segunda estrada o camino cubierto (Fig. 12).

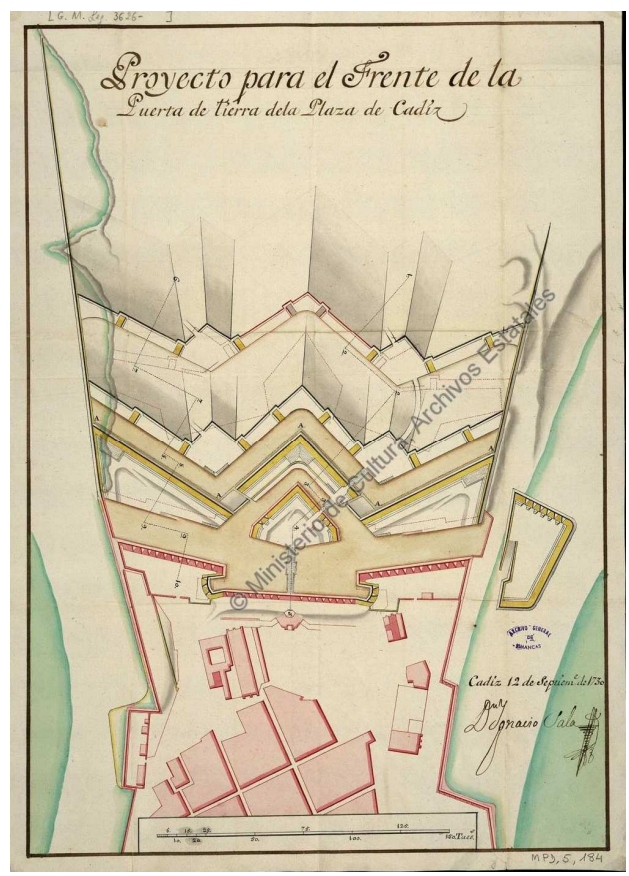

Fig. 11. Proyecto para el Frente de la Puerta de Tierra de la Plaza de Cadiz, septiembre 1730, Ignacio Sala (Archivo General de Simancas, MPD-05-184).

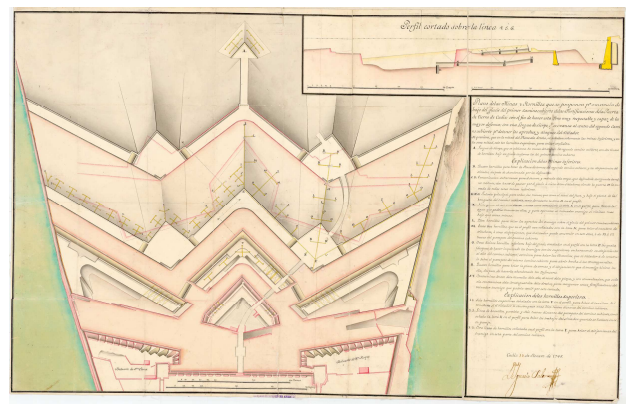

Fig. 12. Plano de las minas y hornillos que se proponen para construir bajo el glacis del primer camino cubierto de las fortificaciones de la Puerta de Tierra de Cadiz, enero 1745, Ignacio Sala (Centro Geográfico del Ejército, Ar.G-T.8-C.3-684).

En enero de 1748, las tres contraguardias se encontraban prácticamente concluidas, y la muralla de la obra coronada, aunque ruinosa, permanecía intacta (Fig. 13). Su derribo y soterrado, bajo el glacis de la primera estrada cubierta, tuvo lugar durante ese año y el siguiente (Fig. 14), si bien la configuración definitiva del glacis no se concluyó hasta el año 1753.

\section{Conclusiones}

Concebida desde sus inicios como un elemento prioritario para el perfeccionamiento del sistema defensivo de la ciudad, la obra coronada del frente de Puerta Tierra de Cádiz, resultó, desde su conclusión, en 1671, una obra mal lograda.

No fue la contienda su principal enemigo, ni la escasez de altura y pendiente de sus terraplenes. Tampoco, la falta de cobertura de sus flancos o la calidad constructiva de sus fundamentos. Fue el temporal de levante y las arenas acumuladas en las dunas del arrecife, sobre el que se edificó, quienes evidenciaron sus carencias y la dejaron, quizás demasiado pronto, fuera de combate.

En los albores del siglo XVIII, la floreciente Cádiz vió desvanecer de nuevo su principal anhelo -contar con una cerca perimetral amurallada- tras el asalto angloholandés de 1596.

Durante la primera mitad del siglo XVIII, el grueso de recursos y esfuerzos fueron destinados a remodelar el sistema defensivo del frente de Puerta Tierra, único punto de acceso terrestre a la ciudad.

El proyecto orginal de remodelación, madurado en plena la Guerra de Sucesión al trono español, fue rescatado por Jorge Prospero Verboom y objeto de numerosas reformulaciones y adaptaciones. Las dificultades técnicas y el elevado costo de las obras, prolongaron su ejecución hasta bien entrada la segunda mitad del siglo, pese a lo cual nunca llegó a ser una obra acabada.

\section{Notas}

1 A.G.S., MPD-08-231, MPD-08-233 у MPD08-247.

2 A.G.S., MPD-53-058, MPD-53-060 у MPD53-064. 


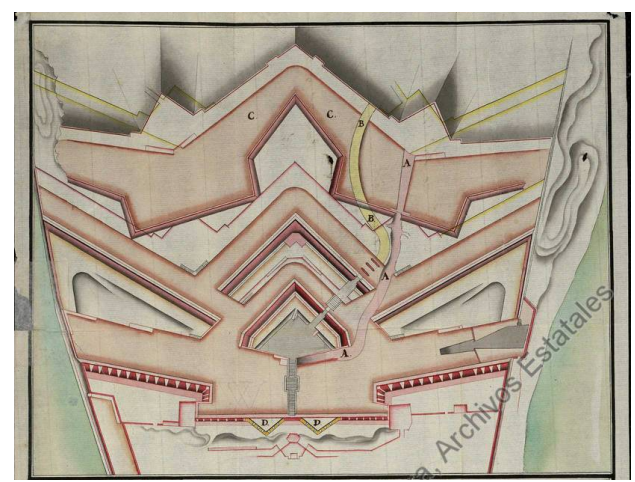

Fig. 13. Plano del frente de Tierra de la Plaza de Cadiz, enero 1748, Ignacio Sala (Archivo General de Simancas, MPD-56-028).

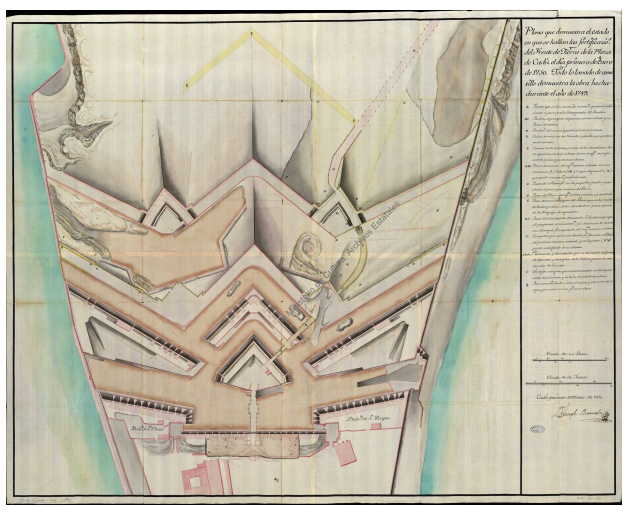

Fig. 14. Plano del frente de la Puerta de Tierra, enero 1750, Joseph Barnola (Archivo General de Simancas, MPD-53-034).

\section{Bibliography}

Abreu, P. de. (1866). Historia del saqueo de Cádiz por los ingleses en 1596, Ayuntamiento de Cádiz (Revista Médica).

Bonet Correa, A. (1991). Cartografía Militar de Plazas Fuertes y Ciudades Españolas. Siglos XVII-XIX. Planos del Archivo Militar Francés, Ministerio de Cultura, Dirección Gral. de Bellas Artes y Archivos, Instituto de Conservación y Restauración de Bienes Culturales, Madrid.

Bustos Rodríguez, M. (2008). "La topografía urbana del Cádiz moderno y su evolución”, Revista AtlánticaMediterránea de Prehistoria y Arqueología Social, 10, pp. 413-444.

Cano-Révora, M.G. (1994). Cádiz y el Real Curepo de Ingenieros Militares (1697-1845), Servicio de Publicaciones de la Universidad de Cádiz, Cádiz.

Capel-Sáez, H., et al. (1983). Los Ingenieros militares en España, siglo XVIII: repertorio biográfico e inventario de su labor científica y espacial, Universitat de Barcelona, Barcelona.

Fernández Álvarez, M. (1966). La España del Emperador Carlos V (1500-1558, 1517-1556), Madrid.

Fernández Cano, V. (1973). Las defensas de Cádiz en la Edad Moderna, Escuela de Estudios Hispano-Americanos de Sevilla, Sevilla.

Ruiz Nieto-Guerrero, M.P. (1999). Historia Urbana de Cádiz. Génesis y formación de una ciudad moderna, Caja San Fernando, Cádiz.

Usherwood, S.; Usherwood, E. (2001). El saqueo de Cádiz. Versión inglesa del ataque de 1596, según el diario del Mary Rose, Servicio de Publicaciones de la Diputación de Cádiz, Cádiz. 